\title{
Aplicación del método de Rietveld al análisis cuantitativo SiC sinterizado en fase líquida
}

\author{
A.L. ORTIZ1 ${ }^{1}$, F.L. CUMBRERA ${ }^{1}$, F. SÁNCHEZ-BAJO ${ }^{2}$, F. GUIBERTEAU ${ }^{2}$, H. XU³ Y N.P. PADTURE ${ }^{3}$ \\ ${ }^{1}$ Departamento de Física. Facultad de Ciencias. Universidad de Extremadura. Ctra de Elvas s/n. 06071. Badajoz (SPAIN) \\ ${ }^{2}$ Departamento de Electrónica e Ingeniería Electromecánica. Escuela de Ingenierías Industriales. \\ Universidad de Extremadura. Ctra de Elvas s/n. 06071. Badajoz (SPAIN) \\ ${ }^{3}$ Department of Metallurgy and Materials Engineering. Institute of Materials Science, Box U-136. \\ University of Connecticut, Storrs, CT 06269 (USA)
}

\begin{abstract}
La determinación de las fracciones en peso de las fases mediante difracción de rayos X es enormemente complicada en cerámicos a base de $\mathrm{SiC}$ debido al intenso solapamiento entre las reflexiones Bragg de los diferentes politipos. No obstante, el método de Rietveld constituye una herramienta poderosa para resolver este problema. En este trabajo se utiliza el método de Rietveld al objeto de determinar las fracciones en peso de las diferentes fases para una muestra de SiC sinterizado en fase líquida (LPS SiC). Nuestros resultados ponen también de manifiesto la necesidad de incorporar correcciones debidas a orientación preferencial para efectuar un análisis cuantitativo preciso.
\end{abstract}

Palabras claves: Método de Rietveld, difracción de rayos X, análisis cuantitativo, carburo de silicio, sinterización en fase líquida.

Quantitative analysis of liquid-phase-sintered SiC by using the Rietveld method

Accurate X-ray quantitative analysis in SiC-based ceramics is a difficult task owing to the strong overlap among the Bragg reflections of the different polytypes. In relation to this point, the Rietveld method can be used as a powerful tool in order to solve this problem. In this study we have applied this procedure to determine the weight fractions of the phases in a liquidphase-sintered $\mathrm{SiC}$ sample. It is shown that the consideration of preferred orientation effects is also indispensable to obtain the accurate proportion of the phases.

Key words: Rietveld method, X-ray diffraction, quantitative analysis, silicon carbide, liquid phase sintering.

\section{INTRODUCCIÓN}

En las últimas décadas ha existido un gran interés en el desarrollo de cerámicos estructurales para aplicaciones tecnológicas. En este contexto, el $\mathrm{SiC}$ sinterizado en fase líquida (LPS SiC) ha adquirido una gran importancia debido a sus interesantes propiedades mecánicas y su elevada resistencia a la oxidación. La sinterización en fase líquida $(1,2)$ de materiales a base de $\mathrm{SiC}$ consiste en mezclar polvos comerciales de $\mathrm{SiC}$ con aditivos de diferentes óxidos $(3,4)$ y oxinitratos $(5,6)$ que funden durante el proceso de sinterización. La fase líquida permite la unión entre partículas de $\mathrm{SiC}$ facilitando la densificación del material. Este procedimiento presenta ciertas ventajas sobre otros métodos tradicionales, entre las que cabría destacar:

-Permite la obtención de materiales con densidades próximas a la teórica

-La temperatura de trabajo es relativamente baja (1900$2000^{\circ} \mathrm{C}$ )

-Permite la obtención de materiales con formas complejas y grandes tamaños

La inclusión de elementos reforzantes se efectúa in situ durante el proceso de sinterización, evitando la necesidad de una segunda etapa de incorporación. Así, eligiendo apropiadamente el polvo de partida y la duración del proceso de sinterizado se pueden obtener in situ microestructuras con refuerzos en la forma de granos alargados de SiC. Por esta técnica constituye una ruta excelente para la fabricación de cerámicos avanzados a bajo coste.

El SiC presenta diferentes politipos cuya estructura cristalina se basa en la coordinación tetraédrica de átomos de carbono y silicio $(7,8)$. En este trabajo se utiliza la notación de Ramsdell (9) para designar los diferentes politipos, en la forma $\mathrm{nX}$, donde $\mathrm{n}$ es un número entero que representa el número de capas de la secuencia de apilamiento, y $\mathrm{X}$ una letra que caracteriza a las posibles simetrías: cúbica $(C)$, hexagonal $(\mathrm{H})$ y romboédrica $(\mathrm{R})$. Aunque existen más de doscientos politipos diferentes (10), los más habituales son el $3 \mathrm{C}, 2 \mathrm{H}, 4 \mathrm{H}, 6 \mathrm{H}$ y $15 \mathrm{R}$. Es común referirse al 3C-SiC como $\beta$-SiC, mientras que a todos los restantes se les denota como $\alpha-\operatorname{SiC}$.

Los politipos presentan diferencias muy pequeñas en su energía libre. Como consecuencia, los materiales cerámicos a base de $\mathrm{SiC}$ pueden estar formados por una mezcla metaestable de varios politipos. Las energías libres de los politipos $\alpha$-SiC son muy similares entre sí, aunque significativamente inferiores a la del $\beta$-SiC (11). Por lo tanto, la fase metaestable $\beta$-SiC se transforma a politipos $\alpha$-SiC a temperaturas elevadas $\left(>1600^{\circ} \mathrm{C}\right)$.

La estabilidad relativa de los diferentes politipos, así como 
sus condiciones de transformación, son cuestiones que no están todavía suficientemente claras, siendo por ello objeto de investigación en la actualidad. Por otro lado, es un hecho bien conocido que las transformaciones tienen una gran influencia sobre la microestructura del material (12), y por lo tanto, sobre sus propiedades mecánicas. Así pues, resulta de gran interés contar con técnicas de análisis que nos permitan conocer las proporciones de los diferentes polimorfos que coexisten en materiales a base de $\mathrm{SiC}$ (13).

La difracción de polvo de rayos $\mathrm{X}$ es el procedimiento más útil para obtener información cuantitativa en mezclas multifásicas (14). Sin embargo, en cerámicos a base de SiC, la determinación de la composición del material es un problema enormemente complejo debido al fuerte solapamiento entre las reflexiones Bragg de los diferentes politipos. El uso del método de Rietveld (15-17) nos permite resolver este problema. Entre las ventajas que ofrece dicho método destacaremos:

-El ajuste del espectro de difracción completo minimiza el problema del solapamiento entre picos de difracción. Efectivamente, todas las reflexiones correspondientes a las diferentes fases se incluyen explícitamente en el refinamiento, reduciendo así la incertidumbre en los valores de las fracciones en peso.

-El proceso de ajuste permite contemplar la existencia de efectos de orientación preferente $(18,19)$.

-No precisa la utilización de una muestra patrón.

El objetivo de este trabajo es el análisis cuantitativo de una muestra de LPS SiC mediante el método de Rietveld. En la siguiente sección se describirán las técnicas experimentales empleadas tanto en la fabricación del material como en su estudio mediante difracción de rayos $\mathrm{X}$.

\section{PROCEDIMIENTO EXPERIMENTAL.}

El material utilizado en este estudio es una muestra policristalina de $\mathrm{SiC}$ ( $80 \%$ en volumen) con una segunda fase de un granate de itrio y aluminio, $\mathrm{Y}_{3} \mathrm{Al}_{5} \mathrm{O}_{12}$, referido como $\mathrm{YAG}$, $(20 \%$ en volumen) localizada en las uniones triples entre granos de $\mathrm{SiC}$.

Los materiales de partida han sido polvos de $\beta$-SiC, $\mathrm{Y}_{2} \mathrm{O}_{3}$ y $\mathrm{Al}_{2} \mathrm{O}_{3}$ de tamaño submicrométrico (Tabla I). Para este estudio, se han utilizado polvos comerciales de SiC (BF-12, H.C. Starck, Germany ). Para obtener YAG se han mezclado polvos de $\mathrm{Y}_{2} \mathrm{O}_{3}$ (Fine-grade, H.C. Starck, Germany) y $\mathrm{Al}_{2} \mathrm{O}_{3}$ (AKP-30, Sumitomo Chemicals, Japan) en fracción molar 5:3. Los detalles del proceso de elaboración del material se describen en la referencia (20).

La sinterización se efectuó en un horno de grafito a $1950^{\circ} \mathrm{C}$, en atmósfera de argón y a la presión de $1.410^{4}-2.110^{4} \mathrm{~N} / \mathrm{m}^{2}$ (0.14-0.20 atm). La muestra sinterizada fue entonces extraida del horno y, posteriormente, se eliminaron dos milímetros de su superficie. Seguidamente, la muestra fue molida para obtener el polvo para los experimentos de difracción de rayos $X$.

Los experimentos de difracción de rayos $\mathrm{X}$ se han efectuado con un difractómetro de polvo Philips PW-1800 perteneciente al Servicio de Difracción de Rayos X de la Universidad de Extremadura. El equipo dispone de un monocromador secundario de grafito, situado entre la muestra y el detector. Los experimentos se han realizado utilizando la radiación $\mathrm{CuK}_{\alpha}, \lambda=1.54183 \AA$, con un ángulo de salida ("take-off") de $6^{\circ}$, fijando las condiciones de trabajo del generador a $40 \mathrm{kV}$ y $35 \mathrm{~mA}$. Para la obtención de los difractogramas se ha utilizado la técnica de barrido por pasos en el intervalo $2 \theta$ com-
TABLA I. COMPOSICIÓN DEL POLVO DE PARTIDA ANTES DEL SINTERIZADO

\begin{tabular}{cc} 
Material & \% en peso \\
\hline$\beta-\mathrm{SiC}$ & 73.9 \\
\hline $\mathrm{Y}_{2} \mathrm{O}_{3}$ & 14.9 \\
\hline $\mathrm{Al}_{2} \mathrm{O}_{3}$ & 11.2 \\
\hline
\end{tabular}

prendido entre $20 \mathrm{y} 95^{\circ}$, seleccionando un paso angular de $0.05^{\circ} 2 \theta$ y un tiempo de contaje de $5 \mathrm{~s}$.

Los ajustes del espectro mediante el método de Rietveld se realizaron empleando el programa FULLPROF (21). Se escogió como función de perfil la función pseudo-Voigt, incluyéndose en el refinamiento los siguientes parámetros:

- fondo, que se modelizó mediante una función polinómica de cuarto grado

- factores de escala de cada una de las fases

- parámetros instrumentales globales (desplazamiento de la posición de cero del difractómetro y desplazamientos sistemáticos, dependientes de la transparencia y del desalineamiento de la muestra).

- parámetros de red para todas las fases

- parámetros de perfil (parámetros de anchura de Caglioti y factor de partición, $\eta$, de la función pseudo-Voigt)

Inicialmente, el ajuste no incluyó correcciones por orientación preferente. En este caso, se observaron diferencias sistemáticas entre las intensidades observadas y calculadas para las reflexiones Bragg 220 y 105 de las fases 3C-SiC y 6H-SiC, respectivamente. Por esta razón se realizó un segundo ajuste introduciendo la función de orientación preferente de MarchDollase (18). Para la fase 6H, la dirección 105 es muy próxima a la dirección 001, que es la dirección en la que suelen orientarse los cristalitos en compuestos de tipo hexagonal. Sin embargo, la inclusión en el ajuste de una corrección en la dirección 001 condujo a un resultado peor que en el caso de la dirección 105.

En el método de Rietveld la información acerca de las proporciones relativas de las fases se obtiene a partir de los factores de escala determinados en el último ciclo del ajuste, empleando la siguiente expresión (22):

$$
\mathrm{X}_{\mathrm{l}}=\frac{\mathrm{s}_{\mathrm{l}} \mathrm{Z}_{\mathrm{l}} \mathrm{M}_{1} \mathrm{~V}_{1}}{\sum_{\mathrm{j}} \mathrm{s}_{\mathrm{j}} \mathrm{Z}_{\mathrm{j}} \mathrm{M}_{\mathrm{j}} \mathrm{V}_{\mathrm{j}}}
$$

donde $\mathrm{Z}_{1}, \mathrm{M}_{1} \mathrm{y} \mathrm{V}_{1}$ son, para la fase $\mathrm{l}$, el número de moléculas en la celda unidad, el peso molecular y el volumen de la celda unidad, respectivamente, siendo $s_{1}$ el factor de escala. El sumatorio en $\mathrm{j}$ se extiende a todas las fases incluidas en el modelo.

Por otra parte, el grado de acuerdo entre los datos experimentales y el modelo empleado en el método de Rietveld se midió empleando los índices usuales (23)

$$
\begin{aligned}
& \mathrm{R}_{\mathrm{p}}=100 \sum_{\mathrm{i}}\left|\mathrm{y}_{\text {iobs }}-\mathrm{y}_{\text {ical }}\right| / \sum_{\mathrm{i}} \mathrm{y}_{\text {iobs }} \\
& \mathrm{R}_{\mathrm{wp}}=100\left[\sum_{\mathrm{i}} \mathrm{w}_{\mathrm{i}}\left(\mathrm{y}_{\text {iob }}-\mathrm{y}_{\mathrm{cal}}\right)^{2} / \sum_{\mathrm{i}} \mathrm{w}_{\mathrm{i}} \mathrm{y}_{\text {iob }}{ }^{2}\right]^{1 / 2}
\end{aligned}
$$

donde $y_{\text {iob }}$ representa la intensidad observada en la posición i-ésima del difractograma, $\mathrm{y}_{\text {ical }}$ la intensidad calculada, $\mathrm{w}_{\mathrm{i}}$ el peso asignado a la medida (usualmente $\mathrm{w}_{\mathrm{i}}=\mathrm{y}_{\mathrm{iob}}{ }^{-1}$ ) y los sumatorios se extienden a todas las observaciones. 


\section{RESULTADOS Y DISCUSIÓN}

Una cuidadosa inspección del difractograma indica claramente que la muestra LPS $\mathrm{SiC}$ contiene $\alpha-\mathrm{SiC}(4 \mathrm{H}$ y $6 \mathrm{H})$ y YAG. La presencia de $\beta-S i C$ en el material no pudo confirmarse debido al solapamiento intenso de sus picos de difracción con los de los politipos $\alpha$-SiC. Sin embargo, dado que el principal constituyente del polvo de partida es el $\beta$-SiC, éste se incluyó en el modelo utilizado en el ajuste. Las reflexiones Bragg de los politipos $\alpha$-SiC muestran que durante el proceso de sinterización se ha producido la transformación $\beta \rightarrow \alpha$. Es bien conocido que el $\beta$-SiC es inestable a temperatura superiores a $1600^{\circ} \mathrm{C}$; por tanto, el sinterizado a $1950^{\circ} \mathrm{C}$ explica la transformación parcial del $\beta$-SiC a $\alpha-S i C$.

En la figura 1 se muestra el resultado del análisis de Rietveld en la situación en la que no se tuvieron en cuenta las correcciones por orientación preferencial. La figura 2 presenta el resultado correspondiente al caso que incluye las correcciones de textura en las direcciones cristalográficas 220 y 105 de los politipos 3C y 6H, respectivamente. Comparando los gráficos de diferencias de ambas figuras concluimos que la introducción de las correcciones por orientación preferencial conduce a un mejor ajuste entre los espectros observado y calculado. Así, el índice $R_{w p}$ es menor en este último caso (12.9 frente a 13.8).

En la tabla II se presentan las fracciones en peso de las fases en el material obtenidas a partir de los factores de escala así como los coeficientes de corrección de la orientación preferente. Los resultados del análisis cuantitativo muestran una concordancia general entre las proporciones relativas de las fases obtenidas en ambos modelos. Como vemos, la ausencia de correcciones por orientación preferencial produce una sobreestimación del politipo 3C-SiC a costa del $6 \mathrm{H}-\mathrm{SiC}$, mientras que la fracción en peso del $4 \mathrm{H}-\mathrm{SiC}$ no varía significativamente. Esta situación puede explicarse si se considera que las reflexiones Bragg del politipo $4 \mathrm{H}-\mathrm{SiC}$ presentan un menor solapamiento con las del 3C-SiC que las del $6 \mathrm{H}-\mathrm{SiC}$, que se encuentran totalmente solapadas.

Por otra parte, el buen acuerdo entre las fracciones en peso calculadas y las del polvo de partida sirve como indicador de la fiabilidad del método empleado en este trabajo. Efectivamente, la suma de las fracciones en peso de los politipos del SiC es muy similar a la fracción en peso de $\beta$-SiC en el polvo de partida. Puede observarse que la fracción en peso del YAG es consistente con la suma de las fracciones en peso de sus constituyentes en el polvo de partida $\left(\mathrm{Y}_{2} \mathrm{O}_{3} \mathrm{y}\right.$ $\left.\mathrm{Al}_{2} \mathrm{O}_{3}\right)$.

\section{CONCLUSIONES.}

En el presente trabajo se muestra que la aplicación del método de Rietveld nos permite obtener las fracciones en peso de los diferentes politipos presentes en una muestra de $\mathrm{SiC}$ sinterizada con fase líquida. Por otra parte, se hace patente que la necesidad de introducir correcciones por orientación preferente, no sólo para mejorar el ajuste, sino con la finalidad de obtener resultados más precisos.

\section{AGRADECIMIENTOS}

Los autores desean expresar su agradecimiento a la Consejería de Educación y Juventud de la Junta de Extremadura por la financiación recibida a través del proyecto IPR98C016.
TABLA II. FRACCIONES EN PESO, FACTORES DE CORRECCIÓN POR ORIENTACIÓN PREFERENTE E ÍNDICES DE ACUERDO DEL MÉTODO DE RIETVELD. EL ÍNDICE $\chi_{R}^{2}$ REPRESENTA EL CHI-CUADRADO REDUCIDO $\left(=\chi^{2} / \mathrm{N}-\mathrm{P}\right.$, CON N EL NÚMERO DE OBSERVACIONES Y P EL NÚMERO DE PARÁMETROS AJUSTADOS).

\begin{tabular}{|l|c|c|}
\hline Modelo & $\begin{array}{c}\text { Sin orientación } \\
\text { preferencial }\end{array}$ & $\begin{array}{c}\text { Con orientación } \\
\text { preferencial }\end{array}$ \\
\hline $3 \mathrm{C}(\%$ en peso) & $49.0(9)$ & $44.2(6)$ \\
\hline $4 \mathrm{H}(\%$ en peso) & $13.4(6)$ & $13.9(5)$ \\
\hline $6 \mathrm{H}(\%$ en peso) & $11.8(8)$ & $15.3(7)$ \\
\hline YAG (\% en peso) & $25.8(4)$ & $26.6(4)$ \\
\hline $\begin{array}{l}\text { Factor de corrección } \\
\text { (dirección 220 de la fase 3C) }\end{array}$ & - & $0.89(5)$ \\
\hline $\begin{array}{l}\text { Factor de corrección } \\
\text { (dirección 105 de la fase 6H) }\end{array}$ & - & $1.10(6)$ \\
\hline $\mathrm{R}_{\mathrm{p}}$ & 10.9 & 9.4 \\
$\mathrm{R}_{\mathrm{wp}}$ & 13.8 & 12.9 \\
$\mathrm{c}^{2}$ & 4.4 & 3.8 \\
\hline
\end{tabular}

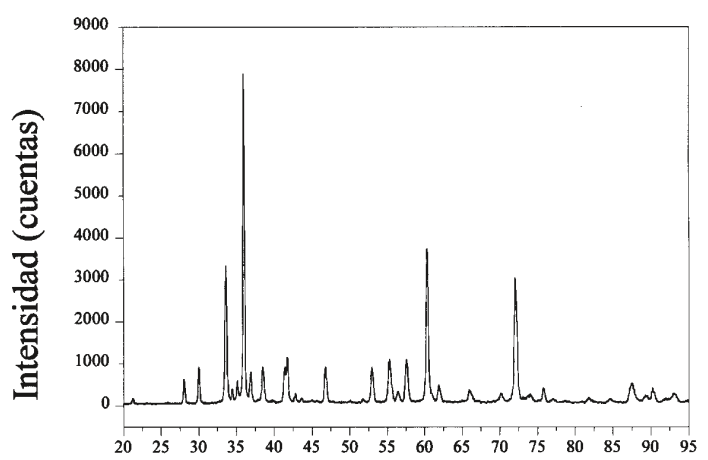

$2 \theta$ (grados)

Figura 1. Espectro de difracción de rayos X de la muestra LPS SiC.

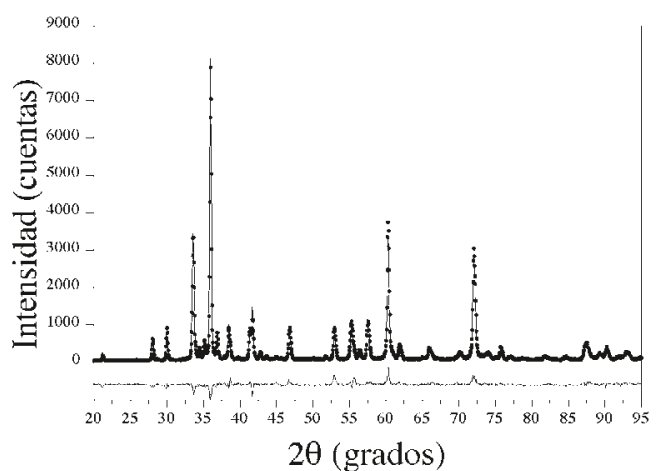

Figura 2. Perfil de difracción observado $(\bullet)$, calculado (-) y diferencia (observado-calculado) en el modelo sin orientación preferencial.

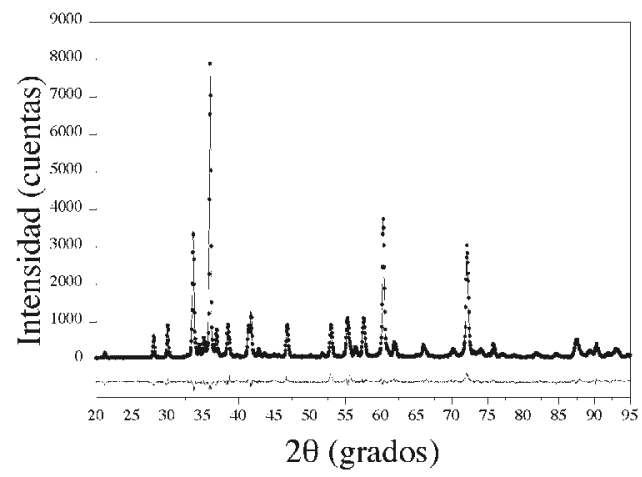

Figura 3. Perfil de difracción observado (•), calculado (-) y diferencia (observado-calculado) en el modelo con orientación preferencial. 


\section{BIBLIOGRAFÍA}

1. J.E. Marion, C.H. Hsueh, A.G. Evans. "Liquid-Phase-Sintering of Ceramics". J. Am. Ceram. Soc. 70 [10] 708-713 (1987).

2. R.M. German. "Liquid Phase Sintering”. Plenum, New York (1985).

3. M.A. Mulla, V.D. Krstic. "Low-Temperature pressureless Sintering of b-Silicon Carbide with Aluminum Oxide and Yttrium Oxide Additions". Am. Ceram. Soc. Bull. 70 [3] 439-443 (1991)

4. L.S. Sigl, H.-J. Kleebe. “Core/Rim structure of liquid-phase-sintered Silicon Carbide". J. Am. Ceram. Soc. 76 [3] 773-776 (1993).

5. J.L. Huang, A.C. Hurford, R.A. Cutler, A.V. Virkar. "Sintering Behavior and properties of SiCAlON Ceramics". J. Matter. Sci. 21 1448-1456 (1986)

6. I. Teusel, C. Russel. "Presureless sintering of Aluminum Nitride/Silicon Carbide Ceramics". J. Matter. Sci. Lett. 11 205-207 (1992).

7. H. Jagodzinski. "Polytypism in SiC crystals". Acta Cryst. 7300 (1954)

8. L.U. Ogbuji. "Origin of Long-Period Polytypism in Polycrystalline SiC". Phys. Stat. Sol. A72 455-461 (1982).

9. L.S. Ramsdell. "Studies in Silicon Carbide". Am. Min. 32 64-82 (1947).

10. P.T.B. Shaffer. "A Review of the Structure of Silicon Carbide". Acta Cryst. B25 477-488 (1969).

11. V. Heine, C. Cheng, G.E. Engel, R.J. Needs. "The Origin of Polytypes in $\mathrm{SiC}$ and $\mathrm{ZnS}^{\prime \prime}$ 507-518 en Wide Band Gap Semiconductors, Material Research Society Symposium Proceedings, vol. 240, Edited by T.D. Moustakas, J.I. Pankove y Y. Hamakawa, Materials Research Society, Pittsburgh, USA (1992).

12. H. Xu. "Microestructural evolution in In-Situ reinforced Silicon Carbide: The effect of starting powder". M.S. Thesis, University of Connecticut (1999).

13. A.L. Ortiz. "Aplicación del método de Rietveld al estudio de la transformación $\beta \rightarrow \alpha$ en policristales de $\mathrm{SiC}$ sinterizados en fase líquida". Tesis de licenciatura, Universidad de Extremadura (1999).

14. H.P. Klug, L.E. Alexander "X-ray diffraction procedures for polycrystalline and amorphous materials" (Chapter 7). John Wiley and Sons, New York (1974).

15. H.M. Rietveld "A profile refinement method for nuclear and magnetic structures". J. Appl. Cryst. 2 65-71 (1969).

16. A. Albinati, B.T.M. Willis "The Rietveld method in Neutron and X-ray Powder Diffraction". J. Appl. Cryst. 15 361-374 (1982).

17. D.L. Bish, S.A. Howard "Quantitative phase analysis using the Rietveld method". J. Appl. Cryst. 21 86-91 (1988).

18. W.A. Dollase "Correction of intensities for preferred orientation in powder diffractometry: application of the March model". J. Appl. Cryst. 19 267-272 (1986).

19. V. Valvoda, M. Chládek, R. Cern. “Joint texture refinement”. J. Appl. Cryst. 29 48-52 (1996)

20. N.P. Padture. "In Situ-Toughened Silicon Carbide”. J. Am. Ceram. Soc. 77 [2] 519-523 (1994).

21. J. Rodríguez-Carvajal "FULLPROF: a program for Rietveld refinement and pattern matching analysis" p. 127 en "Abstracts of the Satellite Meeting on Powder Diffraction of the XV Congress of the IUCr." Toulouse, France. Grenoble, France (1990).

22. R.J.Hill "Expanded use of the Rietveld method in studies of phase abundance in multiphase mixtures". Powder Diffract. 6 [2] 74-77 (1991).

23. R.J. Hill, R.X. Fisher "Profile Agreement Indices in Rietveld and PatternFitting Analysis". J. Appl. Cryst. 23 462-468 (1990). 